\title{
Australian scientists differ on how to attack fraud
}

\section{Sydney}

THE Australian scientific establishment has been thrown into confusion over how to investigate allegations that fraud was committed by a researcher at a Sydneybased research institute.

Accusations were made last December that Dr William McBride, director and founder of Foundation 41, a privately funded teratological institute, fabricated and altered data published in the Australian Journal of Biological Sciences. No one, it seems, is eager to investigate the case.

McBride is well known for his work on the link between the drug thalidomide and birth defects. In June 1982, he claimed in the published paper that orally administered hyoscine, an anti-cholinergic medication, causes birth defects in rabbits. An early draft of the paper appeared as evidence in a federal court trial in Orlando, Florida. The trial, in 1981, involved a lawsuit which alleged that Bendectin, also known as Debendox, contains an anticholinergic drug.

But the two junior scientists who conducted the experiments say that the data are false. According to Mr Phil Vardy, who has retained the original data, McBride added control groups and results, and altered the data to make it appear as though the defects were related to drug dosage. The two scientists say that their names were added to the paper without their knowledge.

McBride claims that the extra rabbits were tested at the University of Virgina. But Norman Swan, producer of the Australian Broadcasting Corporation's science unit, whose programme "The Science Show" first made the allegations, has investigated further. He says that the University of Virginia scientist did not work with rabbits and had probably died at the time the testing occurred.

The matter did not cause comment until the Australian Journal of Biological Sciences, jointly published by the Australian Academy of Science and the Commonwealth Scientific and Industrial Research Organisation (CSIRO), received a second paper from McBride. The paper extended the initial findings and was preceded by a note correcting the original data table; it was published without being refereed.

Vardy and the other scientist involved, Jill French, wrote expressing their reservations and requesting a retraction of their names from the first paper. Their letter was never published. The editor-in-chief, Mr Paul Reekie, says that "the journal will remove the paper from its publication when and if fraud is proven".

It seems that the only avenue to investi-

\section{France moves towards laws on IVF}

\section{Paris}

A working group of the French supreme court (Conseil d'Etat) has just presented its report to the government concerning medical ethics and the law. At the same time, Health Minister Michèle Barzach is publishing a set of decrees aimed at providing legislation controlling in vitro fertilization (IVF) and embryo research. Neither document is yet available from the government stationery office, but the French national daily newspaper Le Monde published extracts on 30 March.

At present, IVF and embryo research is limited only by guidelines published in December 1986 by a national consultative committee on ethics for health and the life sciences. There is no law specifically governing these activities. The ethics committee recommended proscription of IVF not specifically destined to help an infertile couple to have a baby. The committee also recommended the destruction of 'supernumerary' embryos only if the risk to the mother of allowing them to survive is great. Freezing the embryos was thought to be justified, to allow for the failure of embryos to implant in the uterus, but with a maximum shelf-life of 12 months. Regarding embryo research, the committee recommended a moratorium of three years on genetic diagnoses using embryos before implantation. There is no state mechanism to ensure that these guidelines are followed.

The report from the Conseil d'Etat similarly sets out the areas where legislation is necessary, in a broad spectrum of medical practices and experimentation on human subjects. The committee would like any clinical trials that do not have therapeutic aims banned and to ensure that the full and active consent of the participants is given. These concerns are given added weight by the case of Dr Milhaud who performed an anaesthetics experiment on a 'brain-dead' patient without any consent or therapeutic goal.

Barzach aims to limit the abuse of genetic engineering techniques and clinical trials on humans when there are no therapeutic aims. But the decrees have been criticized as a stopgap measure when full legislation is required - and desired by doctors and biologists. The Conseil d'Etat also recommends the drafting of legislation, but Barzach, says Le Monde, feels that this will be a matter of years. gate the case is under the Medical Practitioners' Act which investigates medical, not scientific, improprieties. Foundation 41 says that it has invited an investigation by the Australian Academy of Science. The academy denies this saying it was asked to suggest a committee to look into the 'validity' of McBride's experiments. Jonathan Stone, a University of Sydney professor of anatomy and secretary of the academy, points out that "the issue is not whether the experiments are right or wrong but whether fraud has been committed".

The academy has said that even if invited to investigate the allegations, it would decline to do so, fearing litigation - both against those on the committee and those who present evidence. The National Health and Medical Research Council will not investigate the allegations as they do not support Foundation $41 . \mathrm{Mr}$ Terry Healy, a lawyer with CSIRO, claims that the organization has no legal right to investigate the matter.

The Australian Academy said last week that the only way to deal with cases of scientific fraud is through an independent body with the powers of a royal commission. No procedures at present exist to conduct such an investigation. Tania Ewing

\section{Cyclosporin-yielding fungus found}

New Delhi

THE chance discovery of a fungus at one of the Indian Council of Medical Research (ICMR) institutes has sent waves of excitement through the Indian medical and pharmaceutical community. While screening soil samples for mosquito-killing agents, scientists at the Vector Control Research Centre (VCRC) in Pondicherry have stumbled upon a strain of the fungus Tolypocladium cylindrosporum that yields large quantities of cyclosporin - an immunosuppressive drug widely used for managing patients undergoing organ transplants.

Cyclosporin is manufactured and marketed by Sandoz, which obtains it from the fungus Tolypocladium inflatum. According to VCRC microbiologist Dr K. Balaraman, a secondary metabolite in $T$. cylindrosporum produces an essentially similar product, as three-week-old fungal cultures. Animal studies are soon to begin to test the efficiency of the product which the VCRC is now able to produce in purified crystalline form.

The ICMR is now in the process of identifying an Indian company to undertake commercial production of cyclosporin. Several companies are keen to cash in on the VCRC director discovery which, according to Rajagopalan, "is perhaps the best thing to have happened in the Indian drug scene in recent years." K. S. Jayaraman 